\title{
Resting-State Connectivity Predictors of Response to Psychotherapy in Major Depressive Disorder
}

\begin{abstract}
Andrew Crowther', Moria J Smoski ${ }^{2}$, Jared Minkel ${ }^{2,3}$, Tyler Moore ${ }^{2}$, Devin Gibbs ${ }^{2}$, Chris Petty ${ }^{4}$, Josh Bizzell ${ }^{3,4}$, Crystal Edler Schiller', John Sideris ${ }^{5}$, Hannah Carl ${ }^{6}$ and Gabriel S Dichter*, ${ }^{*}, 2,3,4$

'UNC Neurobiology Curriculum, University of North Carolina at Chapel Hill School of Medicine, Chapel Hill, NC, USA; ${ }^{2}$ Department of Psychiatry and Behavioral Sciences, Duke University Medical Center, Durham, NC, USA; ${ }^{3}$ Department of Psychiatry, University of North Carolina at Chapel Hill School of Medicine, Chapel Hill, NC, USA; ${ }^{2}$ Duke-UNC Brain Imaging and Analysis Center, Duke University Medical Center, Durham, NC, USA; ${ }^{5}$ Frank Porter Graham Child Development Institute, University of North Carolina at Chapel Hill School of Medicine, Chapel Hill, NC, USA; ${ }^{6}$ Department of Psychology and Neuroscience, Duke University, Durham, NC, USA
\end{abstract}

\begin{abstract}
Despite the heterogeneous symptom presentation and complex etiology of major depressive disorder (MDD), functional neuroimaging studies have shown with remarkable consistency that dysfunction in mesocorticolimbic brain systems are central to the disorder. Relatively less research has focused on the identification of biological markers of response to antidepressant treatment that would serve to improve the personalized delivery of empirically supported antidepressant interventions. In the present study, we investigated whether resting-state functional brain connectivity ( $r s-f c M R I$ ) predicted response to Behavioral Activation Treatment for Depression, an empirically validated psychotherapy modality designed to increase engagement with rewarding stimuli and reduce avoidance behaviors. Twenty-three unmedicated outpatients with MDD and 20 matched nondepressed controls completed rs-fcMRI scans after which the MDD group received an average of 12 sessions of psychotherapy. The mean change in Beck Depression Inventory-II scores after psychotherapy was 12.04 points, a clinically meaningful response. Resting-state neuroimaging data were analyzed with a seed-based approach to investigate functional connectivity with four canonical resting-state networks: the default mode network, the dorsal attention network, the executive control network, and the salience network. At baseline, the MDD group was characterized by relative hyperconnectivity of multiple regions with precuneus, anterior insula, dorsal anterior cingulate cortex (dACC), and left dorsolateral prefrontal cortex seeds and by relative hypoconnectivity with intraparietal sulcus, anterior insula, and dACC seeds. Additionally, connectivity of the precuneus with the left middle temporal gyrus and connectivity of the dACC with the parahippocampal gyrus predicted the magnitude of pretreatment MDD symptoms. Hierarchical linear modeling revealed that response to psychotherapy in the MDD group was predicted by pretreatment connectivity of the right insula with the right middle temporal gyrus and the left intraparietal sulcus with the orbital frontal cortex. These results add to the nascent body of literature investigating pretreatment rs-fcMRI predictors of antidepressant treatment response and is the first study to examine rs-fcMRI predictors of response to psychotherapy.
\end{abstract}

Neuropsychopharmacology (2015) 40, 1659-1673; doi: I0.1038/npp.20I5.12; published online 4 February 20I5

\section{INTRODUCTION}

Unipolar major depressive disorder (MDD) is projected to be the second-leading contributor to the global burden of disease across the lifespan by 2020 (Murray and Lopez, 1996) and is currently the leading cause of global burden among all psychiatric and neurological disorders (Collins et al, 2011). The lifetime prevalence rate of MDD is $16 \%$, with an estimated 32-35 million US residents expected to develop the disorder during their lifetimes (Kessler et al, 2003). Despite the societal burden of MDD, there has been

\footnotetext{
*Correspondence: Dr GS Dichter, Department of Psychiatry, University of North Carolina at Chapel Hill School of Medicine, CB 7I55, Chapel Hill, NC 27599-7I55, USA, Tel: +1 9194450132 , Fax: + I 919966 2230, E-mail: dichter@med.unc.edu Received 20 August 2014; revised 24 November 2014; accepted 16 December 2014; accepted article preview online 12 January 2015
}

relatively little progress in improving the efficacy of established antidepressant treatments (Fournier et al, 2010; Undurraga and Baldessarini, 2012): first-line FDA-approved pharmacotherapies demonstrate average response rates of 54 vs 37\% for placebo (Levkovitz et al, 2011), with similar response rates to psychotherapy (Butler et al, 2006; Robinson et al, 1990). Although a number of novel antidepressant agents are currently under development (Murrough and Charney, 2012), one approach to ameliorating the societal burden of MDD is to improve response rates to currently available antidepressant treatments by developing methods to match specific patients to personalized, empirically validated treatments (Kapur et al, 2012; McGrath, et al, 2013; McGrath et al, 2013). To this end, the purpose of the current study was to evaluate rs-fcMRI differences in MDD relative to matched nondepressed controls and to examine whether pretreatment rs-fcMRI predicted response to psychotherapy. 
MDD is characterized by impaired cortico-limbic functioning, including dysfunction in (1) the cortical brain regions that mediate attention, reward-based decision making, and monitoring of emotional salience (Ressler and Mayberg, 2007; Seminowicz et al, 2004); (2) the subcortical brain regions that process affective stimuli (Kumar et al, 2008; Pizzagalli et al, 2009) and that modulate emotional memory formation and retrieval (Dillon et al, 2013); and (3) the coordinated interactions of distributed networks of limbiccortical pathways during processing of cognitive and affective information (Drevets et al, 2008; Northoff et al, 2011). Neuroimaging treatment outcome research has shown that MDD remission is associated with decreased activity in orbitofrontal and medial prefrontal cortex (Goldapple et al, 2004; Kennedy et al, 2007), increased activity in hippocampus and dorsal cingulate cortex (Goldapple et al, 2004), and increased subcortical circuits involved in responses to rewards (Dichter et al, 2009; Stoy et al, 2012) and emotion regulation (Ressler and Mayberg, 2007).

Recent efforts to understand the pathophysiology of MDD have shifted toward a focus on network properties of resting-state brain activity in MDD. As summarized by Mayberg (2007), 'depression is unlikely a disease of a single gene, brain region, or neurotransmitter system. Rather, the syndrome is conceptualized as a systems disorder with a depressive episode viewed as the net effect of failed network regulation' (p. 729). This conceptualization of MDD as a network-level disorder suggests the importance of considering the functional connectivity among subcortical and cortical regions implicated in MDD. More broadly, there has been increased emphasis recently on conceptualizing MDD as a disorder of functional brain connectivity (Wang et al, 2012).

The brain is organized into multiple canonical functional networks, including the default mode network (DMN), the dorsal attention network (DAN), the executive control network (ECN), and the salience network (SN) (Damoiseaux et al, 2006; Fox and Raichle, 2007; Raichle, 2011). The DMN is active and synchronized when the brain is 'at rest' (ie, not engaged with an external task) and is comprised of the posterior cingulate cortex and adjacent precuneus, the medial prefrontal cortex, medial, lateral, and inferior parietal cortex, and medial and inferior temporal cortex (Buckner et al, 2008; Raichle and Snyder, 2007). The DMN supports internal mental activity, (Greicius et al, 2003; Mason et al, 2007) and deactivation of the DMN is associated with goaldirected behaviors (Harrison et al, 2011). Failure to deactivate the DMN is associated with a number of psychiatric disorders, including MDD (Pomarol-Clotet et al, 2008). In contrast to the DMN, the DAN shows increased synchronization during goal-directed processes (Corbetta et al, 1998; Kim, 2010) and includes the intraparietal sulcus/ superior parietal lobule, frontal eye fields, and extrastriate visual areas (Corbetta and Shulman, 2002; Fox et al, 2006). The ECN, including the medial frontal gyrus, superior frontal gyrus, and the anterior cingulate cortex, is engaged during executive function tasks that require cognitive control and working memory (Seeley et al, 2007). The SN, comprised of the anterior insula, the dorsal anterior cingulate cortex (dACC), the amygdala, the substantia nigra/ventral tegmental area, and thalamus (Seeley et al, 2007), segregates internal and external stimuli to guide behavior (Menon and Uddin, 2010; Uddin, 2014) (Some studies have considered the amygdala to be part of the DMN (eg, Sheline et al, 2010). We here consider the amygdala to be part of the SN based on the work of Seely et al (2007) who demonstrated an independent network distinct from the ECN and the DMN that is comprised of the anterior insula, dACC, amygdala, substantia nigra/ventral tegmental area, and thalamus, as well as studies showing anatomical connectivity between the anterior insula and the amygdala (Menon and Uddin, 2010).

A few studies have investigated rs-fcMRI in MDD (for a review, see Wang et al, 2012). Sheline et al (2010) reported hyperconnectivity with bilateral dorsal medial prefrontal cortex across the ECN, the DMN, and the DAN and proposed that these patterns of hyperconnectivity may explain impaired concentration (ECN), increased rumination, selffocus, and vigilance (DMN), and emotional, visceral, and autonomic dysregulation (DAN) in MDD. These findings converge with other reports of aberrant affective network activation at rest and during emotional tasks in MDD (Johansen-Berg et al, 2008; Mayberg et al, 1999; Smoski et al, 2009), DMN hyperactivation during emotional tasks (Sheline et al, 2009), DMN hyperconnectivity at rest in MDD (Lemogne et al, 2009), and increased task-related connectivity (Schlosser et al, 2008; Vasic et al, 2009) and decreased task-related activation (Davidson et al, 2002; Panksepp, 2010) in the ECN in MDD.

To progress the field from cross-sectional studies examining MDD pathophysiology to prospective studies that evaluate potential neuroimaging predictors of response to antidepressant treatment, research is needed that examines baseline neuroimaging measures as putative treatmentspecific biomarkers that predict patient-specific outcomes (Dichter et al, 2012). Indeed, it has been suggested that psychiatric research addressing resting-state network dynamics will be of maximal translational utility if it identifies risk or resilience factors, predicts treatment response or clinical outcomes, or aids in therapeutic targeting (Dietsche et al, 2014; Zhang et al, 2013). Pretreatment rs-fcMRI predictors of antidepressant treatment response appear to be highly contingent on treatment modality, and thus it is noteworthy that no study to date has addressed pretreatment rs-fcMRI predictors of psychotherapy response (Dichter et al, 2015). This omission is notable given that psychotherapy is an empirically validated first-line treatment for MDD (DeRubeis et al, 2008) with comparable efficacy to pharmacological treatment in all but perhaps the most severe cases of MDD (Elkin et al, 1995) and may offer better protection against relapse than pharmacological treatments (Hollon, 2011).

The purpose of the current study was to evaluate rs-fcMRI differences in MDD relative to matched nondepressed controls and to examine whether pretreatment rs-fcMRI predicted response to Behavioral Activation Treatment for Depression (BATD), a structured and validated psychotherapy designed to increase engagement with functional, potentially rewarding behaviors and reduce avoidance behaviors (Hopko et al, 2003). Behavioral activation treatments for MDD are theorized to work by facilitating engagement with potential positive reinforcers and to inhibit the behavioral withdrawal often characteristic of depression (Hopko et al, 2003; Jacobson et al, 2001). We thus focused on the DMN, the DAN, the ECN, and the SN because of linkages between these canonical resting-state networks, 
cognitive and affective processes that are central to the pathophysiology of MDD (Seeley et al, 2007; Sheline et al, 2010; Zhu et al, 2012). We hypothesized cognitive mechanisms of action of BATD. Given the focus of BATD on decreasing avoidance behaviors and increasing behavioral engagement with clinically relevant goals, our strongest hypotheses concerned relations between connectivity in the $\mathrm{SN}$, which has been shown to be over-reactive to negative stimuli in MDD (Hamilton et al, 2012), response to treatment, and anhedonic symptoms specifically.

\section{MATERIALS AND METHODS}

\section{Overview}

The study protocol was approved by the Institutional Review Boards at the University of North Carolina at Chapel Hill and Duke University Medical Centers, and all enrolled participants provided written informed consent. Participants with MDD were recruited via the Cognitive Behavioral Research and Treatment Program at the Duke University Medical Center and nondepressed control participants were recruited via listserves at UNC-Chapel Hill and Duke University. Potential participants completed an initial brief phone screen, and those who passed the phone screen were clinically evaluated, including administration of the Structured Clinical Interview for DSM-IV-TR Axis I Disorders (First et al, 2002) conducted by licensed clinical psychologists or trained clinicians to assess for Axis I disorders, and completed the Hamilton Rating Scale for Depression (HAMD; Hamilton, 1960) and Beck Depression Inventory-2 (BDI; Beck et al, 1996). If still eligible, they were invited to participate in the MRI scan session. Participants with MDD then began psychotherapy by trained and reliable study clinicians. HAMD scores were used to verify inclusion criteria, but only BDI scores are used in analyses.

After their fMRI scans, MDD outpatients received an average of 11.71 ( $\mathrm{SD}=4.08$; range: $2-15)$ weekly sessions of brief BATD. Up to 15 sessions of BATD were offered in this intent-to-treat trial. Early responders were given the option to end therapy after eight sessions, and non-responders received the maximum number of sessions before being referred for additional treatment.

\section{Participants}

Participants in the MDD group met DSM-IV criteria for a current episode of MDD and scored $\geqslant 15$ on the HAMD.
Participants in the control group scored $\leqslant 6$ on the HAMD and did not meet criteria for a current or lifetime episode of a mood disorder. Exclusion criteria included: (1) In the MDD group: current mood, anxiety, psychotic, or substance abuse disorder beyond unipolar MDD or dysthymia, (2) history of psychosis or mania; (3) active suicidal ideation, (4) evidence of organicity, (5) magnetic resonance imaging contraindication (eg, metal in body), (7) history of neurological injury or disease, (8) current pregnancy, or (9) age not between 18 and 50 years. All participants with MDD had been free of psychoactive medication use during the previous month and remained off of psychoactive medications during their participation in this study.

Participants were paid for participating in the clinical assessments and neuroimaging sessions. Twenty-four outpatients with MDD (19 females) and 20 nondepressed controls (14 females) enrolled in the study. One MDD participant was taking antidepressant medication and was excluded from the analyses. Thus the final sample was 23 outpatients with MDD (19 female) and 20 nondepressed controls (14 females). Groups did not differ in age, estimated IQ (measured by the North American Adult Reading Test (Blair and Spreen, 1989), or gender distribution, $p s>0.35$ (see Table 1 for participant characteristics).

\section{Sleepiness}

Given that sleepiness is a common symptom of MDD and recent evidence that a substantial proportion of individuals show a loss of wakefulness during the first $3 \mathrm{~min}$ of restingstate scans (Tagliazucchi and Laufs, 2014), just prior to scans participants completed the Karolinska Sleepiness Scale (KSS; Akerstedt and Gillberg, 1990), a common and well-validated measures of subjective sleepiness that includes a single item with anchor points from 1 (extremely alert) to 9 (extremely sleepy-fighting sleep). Participants with and without MDD reported similar subjective sleepiness: MDD mean $(\mathrm{SD})=5.5(1.4)$; control mean $(\mathrm{SD})=4.6$ $(1.8), t(p)=1.68(0.10)$ (KSS data were not available from two control and three MDD participants). Additionally, participants' eyes were monitored via a camera mounted on the head coil to ensure that participants kept their eyes open throughout the scan session.

\section{Brief BATD}

Behavioral activation treatments have gained increasing interest since the seminal dismantling study of cognitive

Table I Participant Characteristics

\begin{tabular}{|c|c|c|c|c|c|}
\hline & \multicolumn{2}{|c|}{ MDD, $\mathbf{N}=\mathbf{2 3}$} & \multicolumn{2}{|c|}{ Con, $\mathbf{N}=\mathbf{2 0}$} & \multirow[t]{2}{*}{$p$} \\
\hline & Mean (SD) & Range & Mean (SD) & Range & \\
\hline Age (years) & $33.09(7.45)$ & $21-45$ & $31.1(8.82)$ & $20-44$ & 0.443 \\
\hline NAART & | 0.87 (5.34) & $99.2-117.3$ & | | 2.03 (3.83) & $102.6-1 \mid 8.1$ & 0.430 \\
\hline Duration of current episode (months) & $32.96(80.697)$ & $1-384$ & - & - & - \\
\hline
\end{tabular}


behavioral therapy by Jacobson et al (2001) in which behavioral activation appeared equally effective as cognitive therapy in reducing MDD symptoms. At follow-up, behavioral activation was also as effective as cognitive therapy in preventing relapse (Gortner et al, 1998), and a subsequent large-scale randomized trial found that behavioral activation was equivalent to paroxetine in reducing symptoms in moderately to severely depressed individuals (Dimidjian et al, 2006). Recently, Lejuez et al (2001) developed a brief BATD. Although sharing many common elements with previous approaches, BATD is unique in that it is shorter than traditional treatments (only 8-15 sessions) and does not require as extensive skills on the part of the therapist or the patient (Hopko et al, 2003). Treatment proceeds through a series of structured units that (a) provide psychoeducation about MDD and a rationale for the treatment approach; (b) assess and monitor baseline activity levels; (c) develop personally valued goals and a hierarchical plan for goal attainment; and (d) monitor, support, and reward achieving behavioral goals. Preliminary studies have demonstrated that BATD effectively reduces MDD symptoms and is well tolerated in both outpatient (Hopko et al, 2005; Lejuez et al, 2001) and inpatient (Hopko et al, 2003) settings.

\section{Treatment Outcomes}

Treatment outcomes in the MDD group were evaluated by examining changes in BDI scores that were collected at the scan session, every 2 weeks during treatment, and at the last psychotherapy session. BDI scores of $0-13$ indicates minimal MDD, 14-19 indicates mild MDD, 20-28 indicates moderate MDD, and 29-63 indicates severe MDD (Beck et al, 1996). The BDI provides an overall measure of MDD severity and includes items that tap multiple MDD symptom dimensions. Because specific psychological processes are mediated by specific resting-state networks (Raichle, 2011), we examined anhedonia, cognitive, and somatic subscale scores of the BDI in addition to total BDI scores to address whether specific resting-state networks might be predictive of changes in specific MDD constructs. The BDI anhedonia subscale includes items 4, 12, 15, and 21 (Joiner et al, 2003); the cognitive BDI subscale includes items $2,3,5-9$, and 14 (Siegert et al, 2009); and the somatic BDI subscale includes items 1, 4, 10, 11-13, and 15-21 (Siegert et al, 2009). Supplementary analyses evaluated relations between pretreatment rs-fcMRI and psychotherapy motivation, measured by the Situational Motivation Scale (SIMS; Guay et al, 2000), which was also administered every 2 weeks during treatment.

\section{Imaging Methods}

Functional images were acquired at the Duke-UNC Brain Imaging and Analysis Center (BIAC) on a General Electric (Waukesha, WI, USA) MR750 3.0 T scanner equipped with $50 \mathrm{mT} / \mathrm{m}$ gradients $(200 \mathrm{~T} / \mathrm{m} / \mathrm{s}$ slew rate) and an eightchannel head coil for parallel imaging. High-resolution T1weighted anatomical images were acquired with 162 axial slices using a FSPGR pulse sequence $(\mathrm{TR}=7.584 \mathrm{~ms}$; $\mathrm{TE}=$ $2.936 \mathrm{~ms}$; FOV $=256 \mathrm{~mm}$; image matrix $=256 \times 256$; voxel size $=1 \times 1 \times 1 \mathrm{~mm}$; flip angle $=12^{\circ}$ ) and were used for normalization and coregistration with the functional data. This structural image was aligned in a near axial plane defined by the anterior and posterior commissures. Wholebrain functional images were acquired using a spiral-in SENSE sequence $(\mathrm{TR}=1500 \mathrm{~ms} ; \mathrm{TE}=30 \mathrm{~ms} ; \mathrm{FOV}=240$ $\mathrm{mm}$; image matrix, $64 \times 64$; flip angle $=60^{\circ}$; voxel size, $3.75 \times 3.75 \times 4.0 \mathrm{~mm}$; 34 axial slices) to reduce susceptibility artifacts and recover signal in orbital frontal regions (Pruessmann et al, 2001; Truong and Song, 2008). The resting-state functional scan was $300 \mathrm{~s}$ long, and participants were instructed to rest comfortably with their eyes open while viewing a gray fixation cross. A semi-automated high-order shimming program ensured global field homogeneity.

\section{Imaging Data Preprocessing}

The first four volumes of each functional imaging data set were discarded to allow for magnetic field stabilization. Heart rate and respiration were acquired from each participant during the scan, and retrospective correction for physiological motion was performed using AFNI 3dretroicor (Glover et al, 2000), and signal outliers were removed from the data using AFNI 3dDespike. Brain extraction, motion correction, spatial smoothing, and slice-timing correction were then performed using FSL version 5.0.1 (FMRIB Software Library, FMRIB Centre, Oxford University, UK) as previously described (Schiller et al, 2013). Data were affine-registered to MNI152 standard space using MCFLIRT in FSL using an intermodal registration tool (Jenkinson et al, 2002; Smith et al, 2004). Next, white matter and cerebrospinal fluid were regressed out using FMRIB's Automated Segmentation Tool (FAST) in FSL. Voxel-wise temporal autocorrelation was estimated and corrected using FMRIB's Improved Linear Model (Jenkinson and Smith, 2001), and data were bandpass filtered between 0.008 and $0.1 \mathrm{~Hz}$ using custom python scripts. Volumes that exceeded framewise displacement of 0.5 or DVARS (DVARS is a measure of how much the intensity of a brain image changes in comparison to the previous timepoint (Power et al, 2012)) of $0.5 \%$ (mean global intensity of a single volume over brain mask intensity) were removed prior to connectivity analyses (Power et al, 2012). We did not regress global signal intensity (Saad et al, 2012).

\section{Functional Connectivity Analysis}

Functional connectivity was analyzed via a whole-brain seed-based approach. Standard seed regions were used to analyze each canonical resting-state network (Schmidt et al, 2013; Woodward et al, 2011). These seeds were the anterior insula (Elton and Gao, 2013) and dACC (Seeley et al, 2007) for the SN; the posterior intraparietal sulcus/superior parietal lobule (Schmidt et al, 2013; Vincent et al, 2008) for the DAN; the dorsolateral prefrontal cortex (Elton and Gao, 2013; Seeley et al, 2007; Sheline et al, 2010) for the ECN; and the precuneus and medial prefrontal cortex for the DMN (Sheline et al, 2010). Seed regions were 5-mm spheres with centers as described in Raichle (2011) (see Table 2).

Mean fMRI timeseries were extracted from seed ROIs using FSL fslmeants and analyzed as regressors to identify voxels correlated with seed timeseries for each participant in FSL FEAT as a first-level explanatory variable using a 
Table 2 Centers of $5 \mathrm{~mm}$ Sphere of Seed Regions (From Raichle, 2011)

\begin{tabular}{|c|c|c|c|c|}
\hline \multirow[t]{2}{*}{ Network } & \multirow[t]{2}{*}{ Seed regions } & \multicolumn{3}{|c|}{ MNI coordinates } \\
\hline & & $x$ & $Y$ & $z$ \\
\hline \multirow[t]{2}{*}{ Default mode } & Precuneus & 0 & -52 & 27 \\
\hline & Medial prefrontal cortex & -1 & 54 & 27 \\
\hline \multirow[t]{2}{*}{ Dorsal attention } & Left intraparietal sulcus & 41 & -39 & 45 \\
\hline & Right intraparietal sulcus & -44 & -39 & 45 \\
\hline \multirow[t]{3}{*}{ Salience } & Left anterior insula & 41 & 3 & 6 \\
\hline & Right anterior insula & -41 & 3 & 6 \\
\hline & Dorsal anterior cingulate cortex & 0 & 21 & 36 \\
\hline \multirow[t]{2}{*}{ Executive control } & Left dorsal lateral prefrontal cortex & 32 & 45 & 30 \\
\hline & Right dorsal lateral prefrontal cortex & -35 & 45 & 30 \\
\hline
\end{tabular}

general linear model approach with FILM prewhitening (Jenkinson et al, 2012). The resulting parameter estimate maps for each participant were entered into group-level analyses calculated by a mixed effects analysis using Bayesian estimation techniques (FILM, Woolrich et al, 2001) to compare MDD and control groups with respect to seed-based connectivity using FMRIB Local Analysis of Mixed Effects (FLAME $1+2$, Beckmann et al, 2003) using $Z$-statistic images cluster thresholded at $Z>2.3$ with a corrected cluster significance threshold of $p<0.05$. This method of cluster correction, implemented via the cluster thresholding option within FSL FEAT, compares each cluster's estimated significance level (from Gaussian random field theory) with the cluster probability threshold to eliminate clusters below this threshold. Average $Z$-scores from clusters with significantly different connectivity between groups were extracted for each participant to examine relations with antidepressant treatment outcomes in the MDD group. Cluster localizations were based on Harvard-Oxford cortical and subcortical structural probabilistic atlases in FSLView v3.1.8.

\section{RESULTS}

\section{Treatment Response}

Table 3 illustrates that BDI total and subscale scores showed a significant decline from pretreatment to posttreatment. The average decline in BDI total scores was 12.04 $(p<0.001)$, a clinically meaningful response (Jacobson and Truax, 1991). Supplementary Materials SI presents correlations between BDI total and subscale scores at pretreatment and posttreatment.

\section{Head Motion}

There were no significant differences between groups with respect to mean relative or absolute displacement across three dimensions, $p$ 's $>0.05$ (control mean (SD) absolute displacement $=0.255(0.241)$; MDD mean (SD) absolute displacement $=0.189(0.146))$, calculated with FSL MCFLIRT.
Table 3 Change in BDI Total and Subscale Scores After Treatment in the MDD Group

\begin{tabular}{|c|c|c|c|c|c|}
\hline & \multicolumn{2}{|c|}{ Pretreatment } & \multicolumn{2}{|c|}{ Posttreatment } & \multirow[t]{2}{*}{$p$} \\
\hline & Mean (SD) & Range & Mean (SD) & Range & \\
\hline BDI total score & $26.04(7.46)$ & $18-44$ & | $4.00(8.40)$ & $0-25$ & $<0.001$ \\
\hline $\mathrm{BDI}$ anhedonia subscale & $4.97(2.05)$ & $3-10$ & $2.58(2.02)$ & $0-8$ & $<0.001$ \\
\hline BDI somatic subscale & I5.06 (4.98) & $7-23$ & $9.36(6.20)$ & $2-22$ & $<0.001$ \\
\hline BDI cognitive subscale & $10.21(3.89)$ & $5-21$ & $5.26(3.89)$ & $0-11$ & $<0.001$ \\
\hline
\end{tabular}

Pretreatment BDI scores were obtained at the scan session; posttreatment BDI scores were obtained at the last therapy session. Posttreatment BDI subscale scores were not available from one MDD participant.

\section{Group Differences in Functional Connectivity}

Table 4 and Figure 1 illustrate clusters with group differences in seed-based functional connectivity. The MDD group was characterized by relatively increased connectivity between: (1) right anterior insula and left visual cortex; (2) left anterior insula and left superior parietal lobule; (3) dACC and left visual cortex; (4) precuneus and left middle temporal gyrus; and (5) left dorsolateral prefrontal cortex and right motor cortex. The MDD group was characterized by relatively decreased connectivity between: (1) right anterior insula and clusters within left and right middle temporal lobes; (2) left anterior insula seed and the left middle temporal lobe; (3) dACC and left parahippocampal gyrus; and (4) left intraparietal sulcus and left orbitofrontal cortex.

Figure 2 illustrates the high degree of overlap in the left middle temporal gyrus between clusters showing differential connectivity in the MDD group (ie, greater connectivity with the precuneus seed and decreased connectivity with right and left anterior insula seeds).

Supplementary Materials SII presents within-group connectivity maps for each seed regions to illustrate the extent to which group differences in connectivity are related to connectivity maps within the control and MDD samples. Supplementary Materials SIII presents between-group results with the exclusion of the four participants who completed fewer than eight therapy sessions and illustrates highly similar patterns of findings.

\section{Relations between Pretreatment Functional Connectivity and Symptom Severity}

Correlation analyses between pretreatment BDI scores and connectivity magnitude in regions that yielded different connectivity between groups (ie, the nine pairs of regions listed in Table 4) indicated significant relations between MDD severity, as measured by total BDI scores, and connectivity between the precuneus and left middle temporal gyrus connectivity (inverse relation) and connectivity between the dACC and the parahippocampal gyrus (direct relation), as illustrated in Figure 3. Correlations with BDI subscale scores were not significant. 
Table 4 Between Group Differences in Connectivity (Cluster-Corrected $p<0.05$ )

\begin{tabular}{|c|c|c|c|c|c|}
\hline \multirow[t]{2}{*}{ Seed (network) } & \multirow[t]{2}{*}{ Region (BA) } & \multicolumn{3}{|c|}{ MNI coordinates } & \multirow[t]{2}{*}{ Peak z-score } \\
\hline & & $x$ & $Y$ & $\mathbf{Z}$ & \\
\hline Right anterior insula (salience network) & Left visual cortex (BAI7) & -2 & -88 & -8 & 3.87 \\
\hline Left anterior insula (salience network) & Left superior parietal lobule (BA7) & -10 & -66 & 58 & 3.97 \\
\hline Left dorsolateral PFC (executive control network) & Right motor cortex (BA4) & 38 & -24 & 60 & 3.91 \\
\hline \multicolumn{6}{|l|}{ Control >MDD } \\
\hline Right anterior insula (salience network) & Left middle temporal lobe (BA2I) & -60 & -44 & 4 & 4.53 \\
\hline Right anterior insula (salience network) & Right middle temporal lobe (BA22) & 48 & -38 & 8 & 4.29 \\
\hline
\end{tabular}

Abbreviation: BA, Brodmann area.

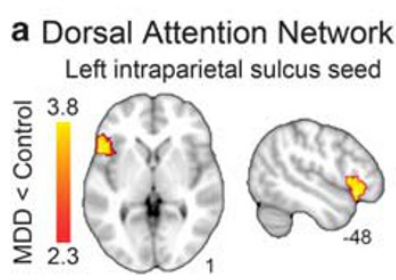

b Default Mode Network

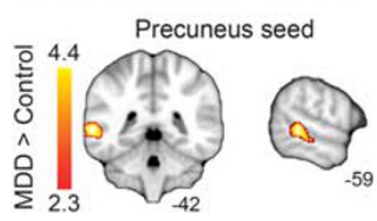

c Executive Control Network

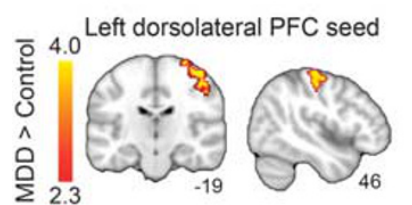

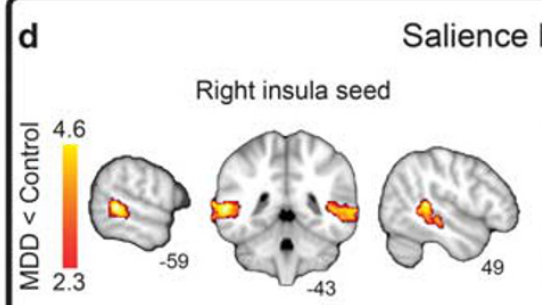
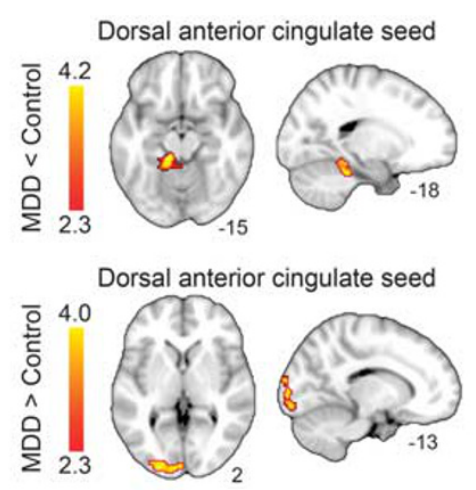

Right insula seed
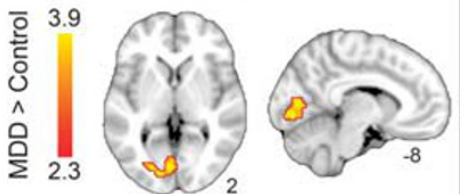

Left insula seed
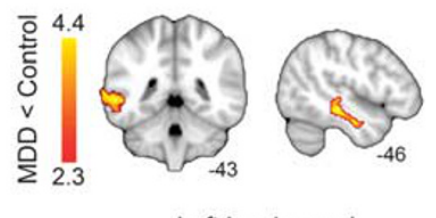

Left insula seed
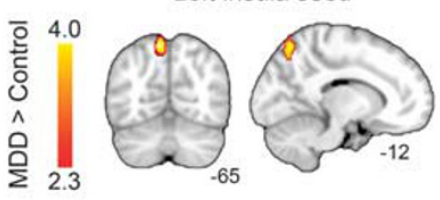

Figure I Group differences in resting-state connectivity in (a) the dorsal attention network (DAN); (b) the default mode network (DMN); (c) the executive control network (ECN); and (d) the salience network (SN). All results are cluster-corrected, $p<0.05$.

\section{Prediction of Treatment Response from Pretreatment Functional Connectivity}

We used hierarchical linear models (HLM), implemented via SAS 'proc mixed' (SAS version 9.3, Cary, NC) to evaluate the predictive effects of pretreatment functional connectivity on response to psychotherapy, as measured by BDI scores (Supplementary Materials SIV present results of correlational analyses testing for relations between baseline
rs-fcMRI values and change in BDI scores, calculated as simple differences between pretreatment and posttreatment BDI scores). HLM models analyzed the capacity of baseline rs-fcMRI data to predict change in symptoms using nine BDI timepoints over 15 weeks. HLM controls for the nonindependence that arises through repeated measures by the inclusion of random effects, and we included random effects for BDI intercept and time. In traditional regression analyses, participants are removed listwise when any of 
their timepoints are missing. In HLM, repeated measures are handled by treating each timepoint for each participant as a separate observation. For this reason, participants with at least one observation (ie, all MDD participants) are included in these analyses.

We used models that tested whether linear change in BDI scores over time was moderated by baseline connectivity included as a time-invariant variable. To address the potential for nonlinear change of time, for all models presented below, we tested models that also included quadratic and cubic effects for time, which were all nonsignificant, indicating that change was indeed linear (all $\mathrm{F}<0.57$, all $p>0.45)$. Models included main effects for time and connectivity plus their interaction terms. Data were centered such that the main effect for connectivity was estimated at time $=0$ (ie, pretreatment), and the main effects for time were estimated at the mean of connectivity values. We examined models separately that assessed the predictive capacity of the nine region pairs that differentiated the MDD and control groups at baseline listed in Table 4 to predict BDI total and BDI anhedonia, cognitive, and

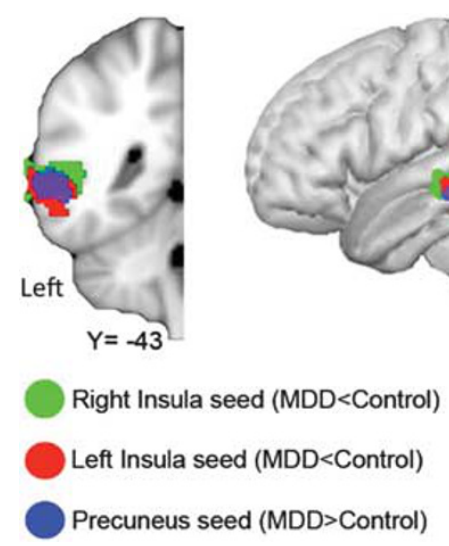

Figure 2 Overlap in the left middle temporal gyrus clusters that demonstrated differential connectivity with the right insula (in green), the left insula (in red), and the precuneus (in blue). somatic subscale scores. Figure 4 illustrates biweekly BDI scores from individual MDD participants and average biweekly BDI scores from all MDD participants. As described earlier, given the focus of BATD on decreasing avoidance behaviors and increasing behavioral engagement with clinically relevant goals, our strongest hypotheses focused on relations between connectivity, response to treatment, and anhedonic symptoms, as well as the severity of MDD symptoms overall. Thus a priori hypotheses focused on BDI total scores and BDI anhedonia subscale scores, and analyses of other BDI subscales should be considered exploratory. Four models yielded significant or marginally significant connectivity $\times$ time interaction effects, as described here:

Prediction of change in BDI anhedonia subscale scores from right insula connectivity with right middle temporal gyrus. Tests of random effects indicated a significant effect for the intercept $(z=2.82 p<0.003)$ and a significant effect for time $(z=1.79, p<0.04)$. The fixed effects indicated a significant negative effect for time $(t=-5.17, p<0.0001)$, indicating that BDI anhedonia subscale scores decreased with time for all participants, as well as for connectivity $(t=2.01, p<0.05)$, indicating that connectivity was associated with BDI anhedonia subscale scores. The critical connectivity $\times$ time interaction was significant $(t=-2.19$, $p<0.04$ ), indicating that varying levels of connectivity were associated with differential change in anhedonia subscale BDI scores over time, and more specifically that the amount of change in BDI anhedonia subscale scores was greatest for those with greater connectivity and least for those with lower connectivity. This effect is illustrated in the top left of Figure 5.

Prediction of change in BDI somatic subscale scores from left intraparietal sulcus connectivity with orbital frontal cortex. Tests of random effects indicated significant effects for the intercept $(z=2.91, p<0.002)$ and for time $(z=1.93, p<0.03)$. The fixed effects indicated a significant negative effect for time $(t=-6.80, p<0.0001)$, indicating that BDI somatic subscale scores decreased with time for all

Relations between pretreatment BDI scores and pretreatment functional connectivity
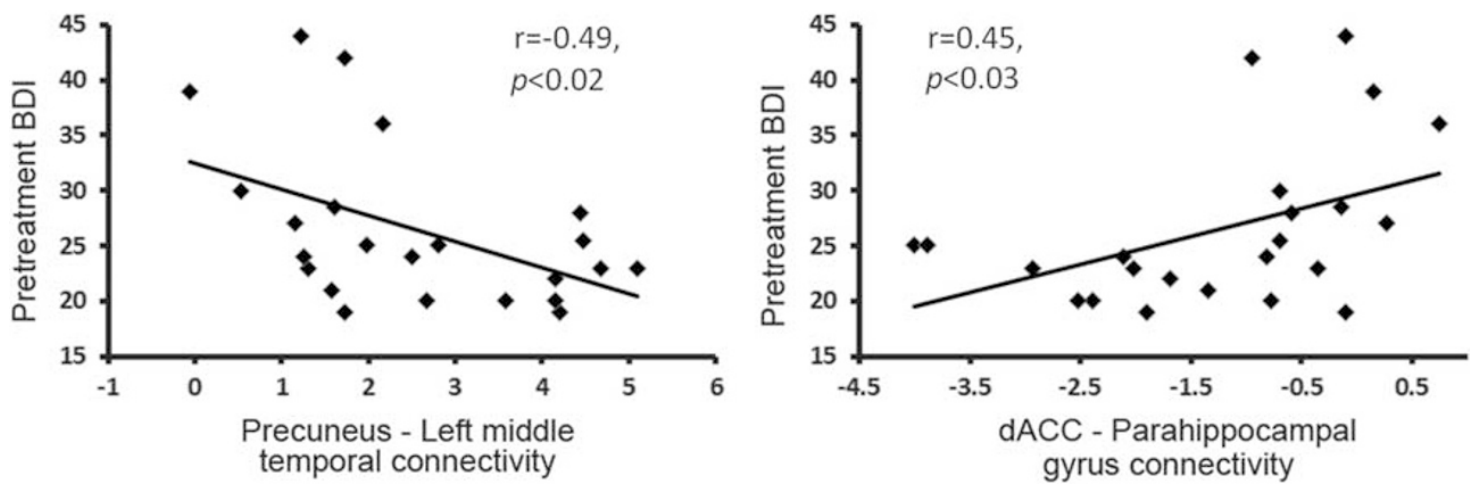

Figure 3 Left: Relations between pretreatment precuneus-left middle temporal gyrus connectivity and pretreatment BDI scores. At pretreatment, the MDD group was characterized by greater precuneus - left middle temporal gyrus connectivity relative to controls. Right: Relations between pretreatment dACC-parahippocampal connectivity and pretreatment BDI scores. At pretreatment, the MDD group was characterized by decreased dACCparahippocampal connectivity relative to controls. 

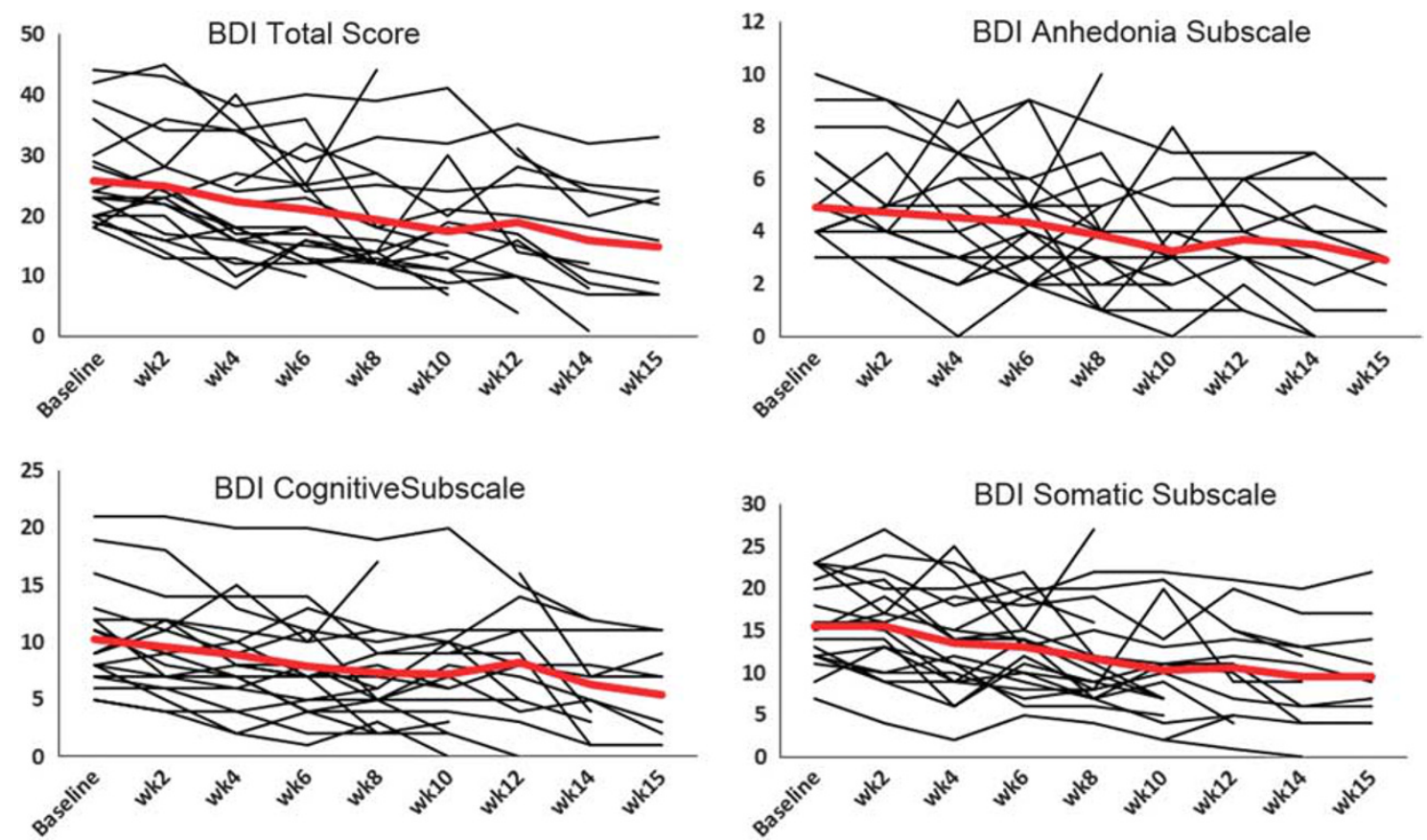

Figure 4 Biweekly BDI scores from individual MDD participants (thin lines) and average biweekly BDI scores from all MDD participants (thick line). Baseline assessments occurred before the start of treatment. Fourteen data points were not available.

$\mathrm{BDI}$ anhedonia scores by time and right insula-right middle temporal gyrus connectivity

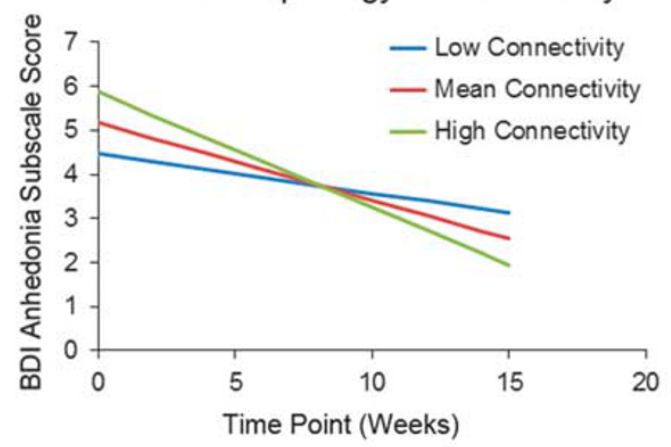

BDI cognitive scores by time and right insula-right middle temporal gyrus connectivity

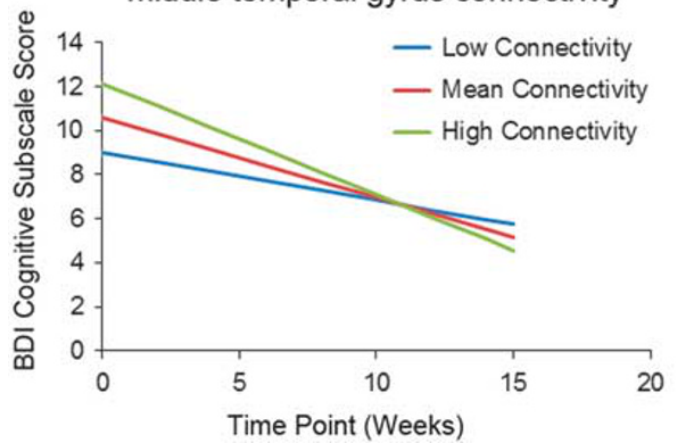

BDI somatic scores by time and left intraparietal sulcus-orbital frontal cortex connectivity

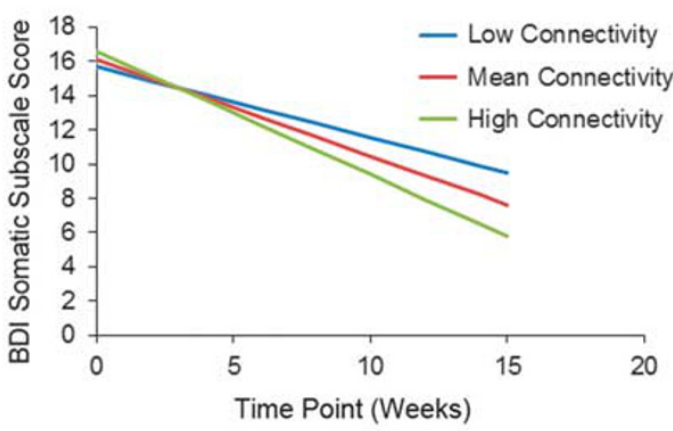

BDI total scores by time and right insula-right middle temporal gyrus connectivity

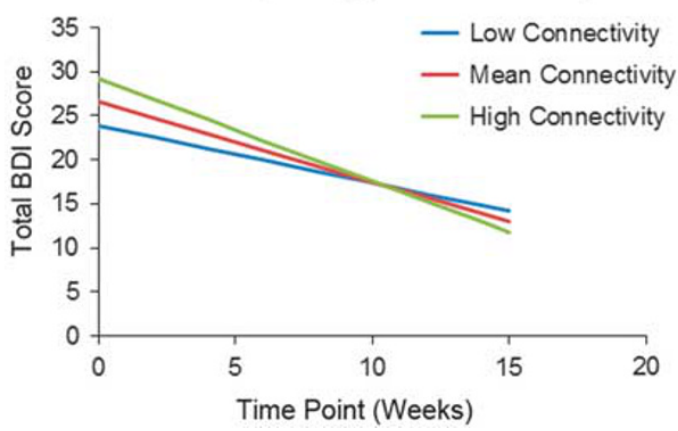

Figure 5 Graphical illustration of the significant interaction between baseline connectivity and time predicting change in BDI scores from the HLM models. The lines represent the range of variability on connectivity: 'low' represents the expectation for change in an individual who is a SD below the mean, 'mean' for someone at the average, and 'high' for someone a SD above. Note that the lines are model-based estimates and do not represent averages but rather ranges of brain connectivity variability. 
participants, but no effect for connectivity $(t=0.51$, $p>0.60)$, indicating that connectivity was not associated with BDI somatic subscale scores. The critical connectivity $\times$ time interaction was marginally significant ( $t=-1.88, p=0.06)$, indicating that varying levels of connectivity were associated with differential change in BDI somatic subscale scores over time, and more specifically that the amount of change in BDI somatic subscale scores was greatest for those with greater connectivity and least for those with lower connectivity. This effect is illustrated in the top right of Figure 5.

Prediction of change in BDI cognitive subscale scores from right insula connectivity with right middle temporal gyrus. Tests of random effects indicated a significant effect for the intercept $(z=2.98, p<0.002)$ and a marginally significant effect for time $(z=1.53, p<0.07)$. The fixed effects indicated a significant negative effect for time $(t=-7.15, p<0.0001)$, indicating that BDI cognitive subscale scores decreased with time for all participants, as well as for connectivity $(t=2.09, p<0.04)$, indicating that connectivity was associated with BDI cognitive subscale scores. The critical connectivity $\times$ time interaction was significant $(t=2.55, p<0.02)$, indicating that varying levels of connectivity were associated with differential change in BDI cognitive subscale scores over time, and more specifically that the amount of change in BDI cognitive subscale scores was greatest for those with greater connectivity and least for those with lower connectivity. This effect is illustrated in the bottom left of Figure 5 .

Prediction of change in total BDI scores from right insula connectivity with right middle temporal gyrus. Tests of random effects indicated significant effects for the intercept $(z=2.89, p<0.002)$ and for time $(z=1.86, p<0.04)$. The fixed effects indicated a significant negative effect for time $(t=-6.99, p<0.0001)$, indicating that BDI scores decreased with time for all participants, and a marginal positive effect for connectivity $(t=1.81, p=0.075)$, indicating that connectivity was marginally positively associated with BDI scores. The critical connectivity $\times$ time interaction was marginally significant $(t=1.80, p=0.074)$, indicating that varying levels of connectivity were associated with differential change in BDI scores over time, and more specifically that the amount of change in BDI scores was greatest for those with greater connectivity and least for those with lower connectivity. This effect is illustrated in the bottom right of Figure 5 .

\section{Prediction of Change in SIMS Scores from Pretreatment rs-fcMRI}

Supplementary Materials SV presents HLM models that predict changes in SIMS scores (Guay et al, 2000) during the course of psychotherapy from rs-fcMRI.

\section{DISCUSSION}

This purpose of this study was to examine differences in resting-state functional connectivity between outpatients with MDD and matched control participants and to investigate whether pretreatment rs-fcMRI connectivity predicted response to behavioral activation psychotherapy. Specifically, we examined whether groups differed in connectivity with seeds previously identified as network hubs in four canonical resting-state networks relevant to the pathophysiology of MDD: the SN, the DAN, the ECN, and the DMN. We examined response to BATD, a validated psychotherapy designed to increase engagement with functional, potentially rewarding behaviors and to reduce avoidance behaviors (Hopko et al, 2003). This psychotherapy modality has been shown to be equally effective as cognitive psychotherapy (Jacobson et al, 1996) and paroxetine treatment (Dimidjian et al, 2006) in reducing MDD symptoms and as effective as cognitive therapy in preventing MDD relapse (Gortner et al, 1998).

The clinical effectiveness of BATD in the current study was consistent with prior trials (Dichter et al, 2009; Hopko et al, 2003): average BDI scores declined 12.25 points, a clinically meaningful response (Jacobson and Truax, 1991). Nevertheless, there was substantial variability in response (ie, the range of change in BDI scores for individual patients was between -6 and +25 points), highlighting the need to develop methods to match specific patients to empirically validated treatments in order to maximize treatment success (Kapur et al, 2012).

We found that connectivity between nine region pairs differentiated the MDD and control groups. Consistent with a priori hypotheses, a number of findings converged on the $\mathrm{SN}$, a brain network that directs attention to salient stimuli in the external environment (Menon and Uddin, 2010). First, we found evidence of decreased connectivity in the MDD group between the dACC (a SN hub) with the left parahippocampal gyrus. The dACC is part of a distributed attentional network that maintains strong reciprocal interconnections with lateral prefrontal, parietal, and motor areas and is implicated in the modulation of attention by influencing sensory and response selection, conflict monitoring, and error detection (eg, Bush et al, 1999; Carter et al, 1999). More recent formulations of the functions of the dACC stress the evaluative, rather than regulatory, role of this region (see, eg, Botvinick, 2007 for a review), highlighting that the AACC detects internal states indicating a need to strengthen top-down control (Badre and Wagner, 2004). Additionally, the dACC serves to integrate the emotional or motivational relevance of stimuli with attentional functions, due to its connections between the limbic system and sensory processing areas (Mayberg, 1997; Mesulam, 1981). Notably, dACC-parahippocampal connectivity was associated with pretreatment BDI scores within the MDD group. Decreased connectivity in the MDD group between the dACC and the parahippocampal gyrus, a component of the limbic system that is differentially active in MDD in the context of memory tasks (Dietsche et al, 2014), emotional processing (Lai, 2014), and reward tasks (Zhang et al, 2013) suggests aberrant dACC regulatory connectivity with this affective processing region in the MDD group that is predictive of symptom severity, suggesting a mechanistic account of negative interpretation biases that characterize MDD (Lawson and MacLeod, 1999).

Also implicating the $\mathrm{SN}$, the left and right anterior insula seeds showed differential connectivity with clusters in visual cortex, the superior parietal lobule and the middle temporal 
gyrus. The anterior insula is sensitive to salient external stimuli and critical for externally oriented attention and internally oriented or self-related cognitions, and the $\mathrm{SN}$ more broadly functions to identify relevant stimuli to guide goal-oriented behaviors (Menon and Uddin, 2010). Additionally, the anterior insula has been implicated in high-level social cognition (Baumgartner et al, 2009), empathy (Singer, 2006), and compassion for social or psychological pain (Immordino-Yang et al, 2009), as well as categorization of negative information and the experience of emotion more generally (Beatty et al, 2014). Differential anterior insular connectivity dovetails with models of MDD that emphasize altered motivational context to personally relevant stimuli and difficulty engaging in cognitively demanding tasks while ignoring irrelevant, negatively valenced stimuli (Yuen et al, 2014), and the current results suggest that greater anterior insula connectivity facilitated response to $\mathrm{BA}$, despite higher initial anhedonia scores.

It is noteworthy that the MDD group was characterized by differential connectivity of the right anterior insula with visual cortex and left anterior insula with left superior parietal lobule. The visual cortex and the superior parietal lobule are critical components of the visual information processing system, with the superior parietal lobule in particular involved in spatial orientation. Visual recognition circuits have been implicated in studies of relations between rs-fcMRI and response to antidepressant treatments (Guo et al, 2013b; Guo et al, 2012; Wang et al, 2014), though the precise functional role of connectivity between the anterior insula and visual processing regions in antidepressant treatment response remains to be elucidated.

The primary focus of the present study was to investigate pretreatment rs-fcMRI predictors of response to BATD. We evaluated this with hierarchical linear regression that modeled BDI scores at nine time points during the course of treatment and that allowed for the inclusion of participants with missing data. In contrast to an approach that examines response to treatment calculated as a simple pretreatment minus posttreatment scores, this approach models scores during the entire course of treatment, mitigating the effect of outlier BDI scores and captures a more detailed picture of the course of treatment response. Four pretreatment connectivity pairs were found to predict treatment response, and three of these involved the anterior insula. Specifically, (1) change in BDI anhedonia subscale scores were predicted by pretreatment right insula-right middle temporal gyrus connectivity; (2) change in BDI cognitive subscale scores were predicted by pretreatment right insula-middle temporal gyrus connectivity; and (3) change in BDI total scores were marginally predicted by pretreatment right insula-middle temporal gyrus connectivity.

One additional BATD-response predictor was identified: change in BDI somatic subscale scores were predicted by pretreatment left intraparietal sulcus-orbital frontal cortex connectivity. The DAN network shows increased synchronization during goal-directed processes (Corbetta et al, 1998; Kim, 2010), and MDD is characterized by poor performance in cognitive control tasks (Veiel, 1997; Zakzanis et al, 1998) as well as dysfunction in dorsolateral and ventrolateral prefrontal cortex and anterior cingulate cortex during cognitive control tasks (Brody et al, 2001; Rogers et al, 2004). MDD is also characterized by altered orbital frontal cortex activation in the context of processing rewards (Dichter et al, 2012; Smoski et al, 2009), anticipating rewards (Dichter et al, 2012), and while processing sad distracting information (Elliott et al, 2002). Thus our finding of decreased connectivity between a DAN network hub and orbitofrontal gyrus may reflect the well-replicated finding of decreased cognitive control over emotion processing in MDD (Dichter et al, 2009) and prevailing neural models of MDD that highlight decreased modulatory control of prefrontal cortical brain regions over limbic brain regions, particularly in the context of emotion processing and emotion regulation (Johnstone et al, 2007; Joormann and Gotlib, 2010; Ray et al, 2005). Additionally, recent studies investigating response to antidepressant medications in MDD highlight that treatment response is associated with increased connectivity between prefrontal cortical and limbic brain regions, possibly implicating greater inhibitory control over neural circuits that process emotions in positive treatment response (Alexopoulos et al, 2012; Lai and Wu, 2012; Lui et al, 2011; Wu et al, 2011; Yang et al, 2014). The somatic subscale as defined by Siegert et al (2009) encompasses mood (sadness), vegetative (eg, sleep, fatigue), attention/concentration, and motivation-related (interest, energy) symptoms and thus may be especially sensitive to disruptions in circuits that underlie both attention and goal-directed behaviors.

It was striking that of the nine region pairs that differentiated groups, four involved connectivity with the middle temporal gyrus (ie, differential connectivity with the precuneus (DMN), anterior insula $(\mathrm{SN})$, and dACC (SN)). Differential precuneus connectivity in MDD is consistent with previous reports of DMN hyperconnectivity in MDD (Broyd et al, 2009; Sheline et al, 2010). Given that the DMN is most active when the brain is at rest (ie, not engaged in goal-directed tasks), and involved in introspective thought and attention orienting, it has been theorized that differential DMN connectivity may be responsible for the negative rumination states that characterize MDD (Broyd et al, 2009). It is also noteworthy that precuneus-left middle temporal gyrus connectivity inversely predicted the severity of pretreatment symptoms in the MDD sample, suggesting a mechanistic linkage between DMN connectivity and MDD severity.

The MDD group was also characterized by aberrant middle temporal gyrus connectivity with a number of seeds. The temporal lobes have a critical role in the subjective experience of emotion (Beatty et al, 2014), and the posterior and superior portions of the temporal sulcus are critically involved in imitation, social cognition (Grossman and Blake, 2002; Iacoboni, 2005), and mentalizing (Sommer et al, 2014). These findings may suggest differential awareness $(\mathrm{DMN})$, salience $(\mathrm{SN})$, and cognitive control (ECN) of mental states in MDD that may contribute to rumination or impaired planful action based on awareness of one's own mental states. Although the current study did not include measures of these constructs, this interpretation is consistent with prevailing cognitive theories of MDD (eg, Watters and Williams, 2011). Specific to the prediction of treatment response, connectivity between anterior insula and middle temporal gyrus may subserve attention to internal affective states and social comparisons that interacted with cognitive symptoms of MDD (eg, guilt, 
worthlessness). In addition, this circuit may also impact the perceived salience of goals and awareness of one's progress toward those goals, interacting with anhedonic symptoms of MDD.

Also of note is that all of the connectivity pairs that predicted better treatment response were consistent with patterns of connectivity closer to that observed in controls. In each case, the region pairs that demonstrated hypoconnectivity relative to the control group and relative hyperconnectivity within the MDD group predicted better treatment response. Contrary to what one might expect, this relatively normalized connectivity was also associated with greater symptom severity in anhedonic and cognitive symptoms of MDD at baseline. This suggests that hypoconnectivity between anterior insula and middle temporal gyrus may serve a compensatory function, and individuals with MDD who do not demonstrate compensatory pretreatment hypoconnectivity may be more amenable to other forms of remediation. Clinically, it is promising to note that greater severity of anhedonic and cognitive symptoms were also associated with greater response, suggesting that treatments such as BATD may be especially useful in targeting individuals with these symptom clusters, although the fact that these patients also had higher BDI total scores suggests the possibility that more severely depressed patients benefited the most from BATD.

The current study has a number of limitations to be addressed in future research. First, a comparison treatment condition (eg, another psychotherapy modality, psychopharmacologic treatment, or magnetic stimulation treatment) was not included, and thus it is not possible to attribute findings specifically to BATD psychotherapy relative to alternative treatments. Indeed, pretreatment rs-fcMRI predictors of antidepressant treatment response appear to be highly contingent on treatment modality (Dichter et al, 2015). Additionally, there were no group differences in connectivity with the DLPFC, a hub of the ECN. The ECN is active during executive function tasks, such as maintaining attention to salient stimuli (Seeley et al, 2007), and recent reports highlight hyperconnectivity of dorsal medial prefrontal cortex with multiple brain networks in MDD (Sheline et al, 2010). Future studies with larger samples will be needed to address the replicability of altered ECN connectivity in MDD. Undoubtedly, robust algorithms predicting treatment response will need to incorporate genetic testing (Frieling and Tadic, 2013), pharmacogenetics (de Leon, 2009), and neuroendocrine function (Holsboer, 2000). It is also well documented that a number of patient factors predict response to antidepressant treatment across treatment modalities, including disease severity, longer duration and frequency of the episodes, comorbid anxiety disorders, and an older age of onset (Kemp et al, 2008). Moreover, longitudinal clinical followup data would be critical to assess relapse after BATD termination as well as rs-fcMRI predictors of relapse, and posttreatment resting-state scans would be needed to address potential mechanisms of antidepressant action on canonical resting-state brain networks. Finally, although a priori hypotheses focused on BDI total and anhedonia subscale scores, findings from other BDI subscales were exploratory in nature and thus should be interpreted with caution until replicated.
In summary, the present study found that MDD is characterized by rs-fcMRI differences in connectivity with a number of canonical brain network hubs. Of note, a number of findings highlight altered connectivity with middle temporal gyrus, a brain area that is involved in imitation, social cognition, and mentalizing, implicating brain regions that code for awareness, salience, and cognitive control of mental states in the pathophysiology of MDD. Additionally, pretreatment connectivity of the anterior insula and intraparietal sulcus predicted response to psychotherapy, a pattern of results consistent with other reports of pretreatment anterior insula metabolism (McGrath et al, 2013) and connectivity (Downar et al, 2013) as predictors of response to psychotherapy and parietal lobe connectivity predicting response to antidepressant medication treatment (Guo et al, 2013a; Wu et al, 2011). More generally, the present study adds to the nascent but growing body of evidence linking pretreatment neuroimaging endophenotypes to antidepressant treatment outcomes (McGrath et al, 2013; McGrath et al, 2013). This line of research has the ultimate goal of improved response rates to currently available antidepressants through matching patients to specific, empirically validated treatment options (Kapur et al, 2012).

\section{FUNDING AND DISCLOSURE}

The project was funded by R21 MH094781 and R21 MH094781 S1 to GSD and MJS, by NIMH K23 MH087754 to MJS, and by NIMH K23 MH081285 to GSD. CES was supported by the UNC Building Interdisciplinary Careers in Women's Health (BIRCWH) Career Development Program (K12 HD001441) and by R21 MH101409. AC was supported by the UNC Neuroscience Center predoctoral training grant, T32NS007431. The content is solely the responsibility of the authors and does not necessarily represent the official views of the National Institutes of Health. The authors declare no conflict of interest.

\section{ACKNOWLEDGEMENTS}

The authors thank MRI technologists Susan Music, Natalie Goutkin, and Luke Poole for assistance with data acquisition, and BIAC Director Dr Allen Song for assistance with various aspects of this project.

\section{REFERENCES}

Akerstedt T, Gillberg M (1990). Subjective and objective sleepiness in the active individual. Int J Neurosci 52: 29-37.

Alexopoulos GS, Hoptman MJ, Kanellopoulos D, Murphy CF, Lim KO, Gunning FM (2012). Functional connectivity in the cognitive control network and the default mode network in late-life depression. J Affect Disord 139: 56-65.

Badre D, Wagner AD (2004). Selection, integration, and conflict monitoring; assessing the nature and generality of prefrontal cognitive control mechanisms. Neuron 41: 473-487.

Baumgartner T, Fischbacher U, Feierabend A, Lutz K, Fehr E (2009). The neural circuitry of a broken promise. Neuron 64: 756-770. 
Beatty EL, Vartanian O, Muller-Gass A, Robertson JA, Mandel DR, Stergiopoulos S (2014). Neuroanatomical correlates of categorizing emotional valence. Neuroreport (e-pub ahead of print).

Beck AT, Steer RA, Ball R, Ranieri W (1996). Comparison of Beck Depression Inventories -IA and -II in psychiatric outpatients. J Pers Assess 67: 588-597.

Beckmann CF, Jenkinson M, Smith SM (2003). General multilevel linear modeling for group analysis in FMRI. Neuroimage 20: 1052-1063.

Blair JR, Spreen O (1989). Predicting premorbid IQ: a revision of the national adult reading test. Clin Neuropsychol 3: 129-136.

Botvinick MM (2007). Conflict monitoring and decision making: reconciling two perspectives on anterior cingulate function. Cogn Affect Behav Neurosci 7: 356-366.

Brody AL, Barsom MW, Bota RG, Saxena S (2001). Prefrontalsubcortical and limbic circuit mediation of major depressive disorder. Semin Clin Neuropsychiatry 6: 102-112.

Broyd SJ, Demanuele C, Debener S, Helps SK, James CJ, SonugaBarke EJ (2009). Default-mode brain dysfunction in mental disorders: a systematic review. Neurosci Biobehav Rev 33: 279-296.

Buckner RL, Andrews-Hanna JR, Schacter DL (2008). The brain's default network: anatomy, function, and relevance to disease. Ann NY Acad Sci 1124: 1-38.

Bush G, Frazier JA, Rauch SL, Seidman LJ, Whalen PJ, Jenike MA et al (1999). Anterior cingulate cortex dysfunction in attentiondeficit/hyperactivity disorder revealed by fMRI and the Counting Stroop. Biol Psychiatry 45: 1542-1552.

Butler AC, Chapman JE, Forman EM, Beck AT (2006). The empirical status of cognitive-behavioral therapy: a review of meta-analyses. Clin Psychol Rev 26: 17-31.

Carter CS, Botvinick MM, Cohen JD (1999). The contribution of the anterior cingulate cortex to executive processes in cognition. Rev Neurosci 10: 49-57.

Collins PY, Patel V, Joestl SS, March D, Insel TR, Daar AS et al (2011). Grand challenges in global mental health. Nature 475: 27-30.

Corbetta M, Akbudak E, Conturo TE, Snyder AZ, Ollinger JM, Drury HA et al (1998). A common network of functional areas for attention and eye movements. Neuron 21: 761-773.

Corbetta M, Shulman GL (2002). Control of goal-directed and stimulus-driven attention in the brain. Nat Rev Neurosci 3: 201-215.

Damoiseaux JS, Rombouts SA, Barkhof F, Scheltens P, Stam CJ, Smith SM et al (2006). Consistent resting-state networks across healthy subjects. Proc Natl Acad Sci USA 103: 13848-13853.

Davidson RJ, Pizzagalli D, Nitschke JB, Putnam K (2002). Depression: perspectives from affective neuroscience. Annu Rev Psychol 53: 545-574.

de Leon J (2009). Pharmacogenomics: the promise of personalized medicine for CNS disorders. Neuropsychopharmacology 34: $159-172$.

DeRubeis RJ, Siegle GJ, Hollon SD (2008). Cognitive therapy versus medication for depression: treatment outcomes and neural mechanisms. Nat Rev Neurosci 9: 788-796.

Dichter GS, Felder JN, Petty C, Bizzell J, Ernst M, Smoski MJ (2009). The effects of psychotherapy on neural responses to rewards in major depression. Biol Psychiatry 66: 886-897.

Dichter GS, Felder JN, Smoski MJ (2009). Affective context interferes with cognitive control in unipolar depression: an fMRI investigation. J Affect Disord 114: 131-142.

Dichter GS, Gibbs D, Smoski MJ (2015). A systematic review of relations between resting-state functional-MRI and treatment response in major depressive disorder. J Affect Disord 172: 8-17.

Dichter GS, Kozink RV, McClernon FJ, Smoski MJ (2012). Remitted major depression is characterized by reward network hyperactivation during reward anticipation and hypoactivation during reward outcomes. J Affect Disord 136: 1126-1134.
Dichter GS, Sikich L, Song A, Voyvodic J, Bodfish JW (2012). Functional neuroimaging of treatment effects in psychiatry: methodological challenges and recommendations. Int J Neurosci 122: 483-493.

Dietsche B, Backes H, Stratmann M, Konrad C, Kircher T, Krug A (2014). Altered neural function during episodic memory encoding and retrieval in major depression. Hum Brain Mapp 35: 4293-4302.

Dillon DG, Dobbins IG, Pizzagalli DA (2013). Weak reward source memory in depression reflects blunted activation of VTA/SN and parahippocampus. Soc Cogn Affect Neurosci 9: 1576-1583.

Dimidjian S, Hollon SD, Dobson KS, Schmaling KB, Kohlenberg RJ, Addis ME et al (2006). Randomized trial of behavioral activation, cognitive therapy, and antidepressant medication in the acute treatment of adults with major depression. J Consult Clin Psychol 74: 658-670.

Downar J, Geraci J, Salomons TV, Dunlop K, Wheeler S, McAndrews MP et al (2013). Anhedonia and reward-circuit connectivity distinguish nonresponders from responders to dorsomedial prefrontal repetitive transcranial magnetic stimulation in major depression. Biol Psychiatry 76: 176-185.

Drevets WC, Savitz J, Trimble M (2008). The subgenual anterior cingulate cortex in mood disorders. CNS Spectr 13: 663-681.

Elkin I, Gibbons RD, Shea MT, Sotsky SM, Watkins JT, Pilkonis PA et al (1995). Initial severity and differential treatment outcome in the National Institute of Mental Health Treatment of Depression Collaborative Research Program. J Consult Clin Psychol 63: 841-847.

Elliott R, Rubinsztein JS, Sahakian BJ, Dolan RJ (2002). The neural basis of mood-congruent processing biases in depression. Arch Gen Psychiatry 59: 597-604.

Elton A, Gao W (2013). Divergent task-dependent functional connectivity of executive control and salience networks. Cortex 51: $56-66$.

First MB, Spitzer RL, Gibbon M, Williams JBW (2002). Structured Clinical Interview for DSM-IV-TR Axis I Disorders, Research Version, Patient Edition. (SCID-I/P). New York State Psychiatric Institute: Biometrics Research: New York, NY, USA.

Fournier JC, DeRubeis RJ, Hollon SD, Dimidjian S, Amsterdam JD, Shelton RC et al (2010). Antidepressant drug effects and depression severity: a patient-level meta-analysis. JAMA 303: 47-53.

Fox MD, Corbetta M, Snyder AZ, Vincent JL, Raichle ME (2006). Spontaneous neuronal activity distinguishes human dorsal and ventral attention systems. Proc Natl Acad Sci USA 103: 10046-10051.

Fox MD, Raichle ME (2007). Spontaneous fluctuations in brain activity observed with functional magnetic resonance imaging. Nat Rev Neurosci 8: 700-711.

Frieling H, Tadic A (2013). Value of genetic and epigenetic testing as biomarkers of response to antidepressant treatment. Int Rev Psychiatry 25: 572-578.

Glover GH, Li TQ, Ress D (2000). Image-based method for retrospective correction of physiological motion effects in fMRI: RETROICOR. Magn Reson Med 44: 162-167.

Goldapple K, Segal Z, Garson C, Lau M, Bieling P, Kennedy S et al (2004). Modulation of cortical-limbic pathways in major depression: treatment-specific effects of cognitive behavior therapy. Arch Gen Psychiatry 61: 34-41.

Gortner ET, Gollan JK, Dobson KS, Jacobson NS (1998). Cognitivebehavioral treatment for depression: relapse prevention. J Consult Clin Psychol 66: 377-384.

Greicius MD, Krasnow B, Reiss AL, Menon V (2003). Functional connectivity in the resting brain: a network analysis of the default mode hypothesis. Proc Natl Acad Sci USA 100: 253-258.

Grossman ED, Blake R (2002). Brain areas active during visual perception of biological motion. Neuron 35: 1167-1175. 
Guay F, Vallerand R, Blanchard C (2000). On the assessment of situational intrinsic and extrinsic motivation: the Situational Motivation Scale (SIMS). Motiv Emotion 24: 175-213.

Guo WB, Liu F, Xue Z, Gao K, Liu Z, Xiao C et al (2013a). Abnormal resting-state cerebellar-cerebral functional connectivity in treatment-resistant depression and treatment sensitive depression. Prog Neuropsychopharmacol Biol Psychiatry 44: 51-57.

Guo WB, Liu F, Xue Z, Gao K, Liu Z, Xiao C et al (2013b). Decreased interhemispheric coordination in treatment-resistant depression: a resting-state fMRI study. PLoS One 8: e71368.

Guo WB, Liu F, Xue ZM, Xu XJ, Wu RR, Ma CQ et al (2012). Alterations of the amplitude of low-frequency fluctuations in treatment-resistant and treatment-response depression: a resting-state fMRI study. Prog Neuropsychopharmacol Biol Psychiatry 37: 153-160.

Hamilton JP, Etkin A, Furman DJ, Lemus MG, Johnson RF, Gotlib IH (2012). Functional neuroimaging of major depressive disorder: a meta-analysis and new integration of base line activation and neural response data. Am J Psychiatry 169: 693-703.

Hamilton MA (1960). A rating scale for depression. J Neurol Neurosurg Psychiatry 23: 56-62.

Harrison BJ, Pujol J, Contreras-Rodriguez O, Soriano-Mas C, Lopez-Sola M, Deus J et al (2011). Task-induced deactivation from rest extends beyond the default mode brain network. PLoS One 6: e22964.

Hollon SD (2011). Cognitive and behavior therapy in the treatment and prevention of depression. Depress Anxiety 28: 263-266.

Holsboer F (2000). The corticosteroid receptor hypothesis of depression. Neuropsychopharmacology 23: 477-501.

Hopko DR, Bell JL, Armento MEA, Hunt MK, Lejuez CW (2005). Behavior thearpy for depressed cancer patients in primary care. Psychother Theor Res Pract Train 42: 236-243.

Hopko DR, Lejuez CW, Ruggiero KJ, Eifert GH (2003). Contemporary behavioral activation treatments for depression: procedures, principles, and progress. Clin Psychol Rev 23: 699-717.

Iacoboni M (2005). Neural mechanisms of imitation. Curr Opin Neurobiol 15: 632-637.

Immordino-Yang MH, McColl A, Damasio H, Damasio A (2009). Neural correlates of admiration and compassion. Proc Natl Acad Sci USA 106: 8021-8026.

Jacobson NS, Dobson KS, Truax PA, Addis ME, Koerner K, Gollan JK et al (1996). A component analysis of cognitive-behavioral treatment for depression. J Consult Clin Psychol 64: 295-304.

Jacobson NS, Martell CR, Dimidjian S (2001). Behavioral activation treatment for depression: returning to contextual roots. Clin Psychol Sci Pract 8: 255-270.

Jacobson NS, Truax P (1991). Clinical significance: a statistical approach to defining meaningful change in psychotherapy research. J Consult Clin Psychol 59: 12-19.

Jenkinson M, Bannister P, Brady M, Smith S (2002). Improved optimization for the robust and accurate linear registration and motion correction of brain images. Neuroimage 17: 825-841.

Jenkinson M, Beckmann CF, Behrens TE, Woolrich MW, Smith SM (2012). Fsl. Neuroimage 62: 782-790.

Jenkinson M, Smith S (2001). A global optimisation method for robust affine registration of brain images. Med Image Analy 5: 143-156.

Johansen-Berg H, Gutman DA, Behrens TE, Matthews PM, Rushworth MF, Katz E et al (2008). Anatomical connectivity of the subgenual cingulate region targeted with deep brain stimulation for treatment-resistant depression. Cereb Cortex 18: $1374-1383$.

Johnstone T, van Reekum CM, Urry HL, Kalin NH, Davidson RJ (2007). Failure to regulate: counterproductive recruitment of top-down prefrontal-subcortical circuitry in major depression. J Neurosci 27: 8877-8884.
Joiner TE, Brown JS, Metalsky GI (2003). A test of the tripartite model's prediction of anhedonia's specificity to depression: patients with major depression versus patients with schizophrenia. Psychiatry Res 119: 243-250.

Joormann J, Gotlib IH (2010). Emotion regulation in depression: relation to cognitive inhibition. Cogn Emot 24: 281-298.

Kapur S, Phillips AG, Insel TR (2012). Why has it taken so long for biological psychiatry to develop clinical tests and what to do about it? Mol Psychiatry 17: 1174-1179.

Kemp AH, Gordon E, Rush AJ, Williams LM (2008). Improving the prediction of treatment response in depression: integration of clinical, cognitive, psychophysiological, neuroimaging, and genetic measures. CNS Spectr 13: 1066-1086; quiz 1087-1068.

Kennedy SH, Konarski JZ, Segal ZV, Lau MA, Bieling PJ, McIntyre RS et al (2007). Differences in brain glucose metabolism between responders to CBT and venlafaxine in a 16-week randomized controlled trial. Am J Psychiatry 164: 778-788.

Kessler RC, Berglund P, Demler O, Jin R, Koretz D, Merikangas KR et al (2003). The epidemiology of major depressive disorder: results from the National Comorbidity Survey Replication (NCS-R). JAMA 289: 3095-3105.

Kim H (2010). Dissociating the roles of the default-mode, dorsal, and ventral networks in episodic memory retrieval. Neuroimage 50: $1648-1657$.

Kumar P, Waiter G, Ahearn T, Milders M, Reid I, Steele JD (2008). Abnormal temporal difference reward-learning signals in major depression. Brain 131: 2084-2093.

Lai CH (2014). Patterns of cortico-limbic activations during visual processing of sad faces in depression patients: a coordinatebased meta-analysis. J Neuropsychiatry Clin Neurosci 26: 34-43.

Lai CH, Wu YT (2012). Frontal regional homogeneity increased and temporal regional homogeneity decreased after remission of first-episode drug-naive major depressive disorder with panic disorder patients under duloxetine therapy for 6 weeks. J Affect Disord 136: 453-458.

Lawson C, MacLeod C (1999). Depression and the interpretation of ambiguity. Behav Res Ther 37: 463-474.

Lejuez CW, Hopko DR, Hopko SD (2001). A brief behavioral activation treatment for depression. Treatment manual. Behav Modif 25: 255-286.

Lemogne C, le Bastard G, Mayberg H, Volle E, Bergouignan L, Lehericy $S$ et al (2009). In search of the depressive self: extended medial prefrontal network during self-referential processing in major depression. Soc Cogn Affect Neurosci 4: 305-312.

Levkovitz Y, Tedeschini E, Papakostas GI (2011). Efficacy of antidepressants for dysthymia: a meta-analysis of placebocontrolled randomized trials. J Clin Psychiatry 72: 509-514.

Lui S, Wu Q, Qiu L, Yang X, Kuang W, Chan RC et al (2011). Resting-state functional connectivity in treatment-resistant depression. Am J Psychiatry 168: 642-648.

Mason MF, Norton MI, Van Horn JD, Wegner DM, Grafton ST, Macrae CN (2007). Wandering minds: the default network and stimulus-independent thought. Science 315: 393-395.

Mayberg HS (1997). Limbic-cortical dysregulation: a proposed model of depression. J Neuropsychiatry Clin Neurosci 9: 471-481.

Mayberg HS (2007). Defining the neural circuitry of depression: toward a new nosology with therapeutic implications. Biol Psychiatry 61: 729-730.

Mayberg HS, Liotti M, Brannan SK, McGinnis S, Mahurin RK, Jerabek PA et al (1999). Reciprocal limbic-cortical function and negative mood: converging PET findings in depression and normal sadness. Am J Psychiatry 156: 675-682.

McGrath CL, Kelley ME, Dunlop BW, Holtzheimer Iii PE, Craighead WE, Mayberg HS (2013). Pretreatment brain states identify likely nonresponse to standard treatments for depression. Biol Psychiatry 76: 527-535.

McGrath CL, Kelley ME, Holtzheimer PE, Dunlop BW, Craighead WE, Franco AR et al (2013). Toward a neuroimaging treatment 
selection biomarker for major depressive disorder. JAMA Psychiatry 70: 821-829.

Menon V, Uddin LQ (2010). Saliency, switching, attention and control: a network model of insula function. Brain Struct Funct 214: 655-667.

Mesulam MM (1981). A cortical network for directed attention and unilateral neglect. Ann Neurol 10: 309-325.

Murray CJ, Lopez AD (1996). The Global Burden of Disease: A Comprehensive Assessment of Mortality and Disability from Disease, Injuries, and Risk Factors in 1990 and Projected to 2020 (Vol. 1) Harvard University press: Cambridge, MA, USA.

Murrough JW, Charney DS (2012). Is there anything really novel on the antidepressant horizon? Curr Psychiatry Rep 14: 643-649.

Northoff G, Wiebking C, Feinberg T, Panksepp J (2011). The 'resting-state hypothesis' of major depressive disorder-a translational subcortical-cortical framework for a system disorder. Neurosci Biobehav Rev 35: 1929-1945.

Panksepp J (2010). Affective neuroscience of the emotional BrainMind: evolutionary perspectives and implications for understanding depression. Dialogues Clin Neurosci 12: 533-545.

Pizzagalli D, Holmes A, Dillon D, Goetz E, Birk J, Bogdan R et al (2009). Reduced caudate and nucleus accumbens response to rewards in unmedicated individuals with major depressive disorder. Am J Psychiatr 166: 702-710.

Pomarol-Clotet E, Salvador R, Sarro S, Gomar J, Vila F, Martinez A et al (2008). Failure to deactivate in the prefrontal cortex in schizophrenia: dysfunction of the default mode network? Psychol Med 38: 1185-1193.

Power JD, Barnes KA, Snyder AZ, Schlaggar BL, Petersen SE (2012). Spurious but systematic correlations in functional connectivity MRI networks arise from subject motion. Neuroimage 59: 2142-2154.

Pruessmann KP, Weiger M, Bornert P, Boesiger P (2001). Advances in sensitivity encoding with arbitrary k-space trajectories. Magn Reson Med 46: 638-651.

Raichle ME (2011). The restless brain. Brain Connect 1: 3-12.

Raichle ME, Snyder AZ (2007). A default mode of brain function: a brief history of an evolving idea. Neuroimage 37: 1083-1090 discussion 1097-1089.

Ray RD, Ochsner KN, Cooper JC, Robertson ER, Gabrieli JD, Gross JJ (2005). Individual differences in trait rumination and the neural systems supporting cognitive reappraisal. Cogn Affect Behav Neurosci 5: 156-168.

Ressler KJ, Mayberg HS (2007). Targeting abnormal neural circuits in mood and anxiety disorders: from the laboratory to the clinic. Nat Neurosci 10: 1116-1124.

Robinson LA, Berman JS, Neimeyer RA (1990). Psychotherapy for the treatment of depression: a comprehensive review of controlled outcome research. Psychol Bull 108: 30-49.

Rogers MA, Kasai K, Koji M, Fukuda R, Iwanami A, Nakagome K et al (2004). Executive and prefrontal dysfunction in unipolar depression: a review of neuropsychological and imaging evidence. Neurosci Res 50: 1-11.

Saad ZS, Gotts SJ, Murphy K, Chen G, Jo HJ, Martin A et al (2012). Trouble at rest: how correlation patterns and group differences become distorted after global signal regression. Brain Connect 2: 25-32.

Schiller CE, Minkel J, Smoski MJ, Dichter GS (2013). Remitted major depression is characterized by reduced prefrontal cortex reactivity to reward loss. J Affect Disord 151: 756-762.

Schlosser RG, Wagner G, Koch K, Dahnke R, Reichenbach JR, Sauer $H$ (2008). Fronto-cingulate effective connectivity in major depression: a study with fMRI and dynamic causal modeling. Neuroimage 43: 645-655.

Schmidt SA, Akrofi K, Carpenter-Thompson JR, Husain FT (2013). Default mode, dorsal attention and auditory resting state networks exhibit differential functional connectivity in tinnitus and hearing loss. PLoS One 8: e76488.
Seeley WW, Menon V, Schatzberg AF, Keller J, Glover GH, Kenna $\mathrm{H}$ et al (2007). Dissociable intrinsic connectivity networks for salience processing and executive control. J Neurosci 27: 2349-2356.

Seminowicz DA, Mayberg HS, McIntosh AR, Goldapple K, Kennedy S, Segal Z et al (2004). Limbic-frontal circuitry in major depression: a path modeling metanalysis. Neuroimage 22: 409-418.

Sheline YI, Barch DM, Price JL, Rundle MM, Vaishnavi SN, Snyder AZ et al (2009). The default mode network and self-referential processes in depression. Proc Natl Acad Sci USA 106: 1942-1947.

Sheline YI, Price JL, Yan ZZ, Mintun MA (2010). Resting-state functional MRI in depression unmasks increased connectivity between networks via the dorsal nexus. Proc Natl Acad Sci USA 107: 11020-11025.

Siegert RJ, Walkey FH, Turner-Stokes L (2009). An examination of the factor structure of the Beck Depression Inventory-II in a neurorehabilitation inpatient sample. J Int Neuropsychol Soc 15: 142-147.

Singer T (2006). The neuronal basis and ontogeny of empathy and mind reading: review of literature and implications for future research. Neurosci Biobehav Rev 30: 855-863.

Smith SM, Jenkinson M, Woolrich MW, Beckmann CF, Behrens TEJ, Johansen-Berg $\mathrm{H}$ et al (2004). Advances in functional and structural MR image analysis and implementation as FSL. Neuroimage 23(Suppl 1): S208-S219.

Smoski MJ, Felder J, Bizzell J, Green SR, Ernst M, Lynch TR et al (2009). fMRI of alterations in reward selection, anticipation, and feedback in major depressive disorder. J Affect Disord 118: 69-78.

Sommer M, Meinhardt J, Rothmayr C, Dohnel K, Hajak G, Rupprecht $\mathrm{R}$ et al (2014). Me or you? Neural correlates of moral reasoning in everyday conflict situations in adolescents and adults. Soc Neurosci 9: 452-470.

Stoy M, Schlagenhauf F, Sterzer P, Bermpohl F, Hagele C, Suchotzki K et al (2012). Hyporeactivity of ventral striatum towards incentive stimuli in unmedicated depressed patients normalizes after treatment with escitalopram. J Psychopharmacol 26: 677-688.

Tagliazucchi E, Laufs H (2014). Decoding wakefulness levels from typical fMRI resting-state data reveals reliable drifts between wakefulness and sleep. Neuron 82: 695-708.

Truong TK, Song AW (2008). Single-shot dual-z-shimmed sensitivity-encoded spiral-in/out imaging for functional MRI with reduced susceptibility artifacts. Magn Reson Med 59: 221-227.

Uddin LQ (2014). Salience processing and insular cortical function and dysfunction. Nat Rev Neurosci 16: 55-61.

Undurraga J, Baldessarini RJ (2012). Randomized, placebocontrolled trials of antidepressants for acute major depression: thirty-year meta-analytic review. Neuropsychopharmacology 37: 851-864.

Vasic N, Walter H, Sambataro F, Wolf RC (2009). Aberrant functional connectivity of dorsolateral prefrontal and cingulate networks in patients with major depression during working memory processing. Psychol Med 39: 977-987.

Veiel HO (1997). A preliminary profile of neuropsychological deficits associated with major depression. J Clin Exp Neuropsychol 19: 587-603.

Vincent JL, Kahn I, Snyder AZ, Raichle ME, Buckner RL (2008). Evidence for a frontoparietal control system revealed by intrinsic functional connectivity. J Neurophysiol 100: 3328-3342.

Wang L, Hermens DF, Hickie IB, Lagopoulos J (2012). A systematic review of resting-state functional-MRI studies in major depression. J Affect Disord 142: 6-12.

Wang L, Kuang WH, Xu JJ, Lei D, Yang YC (2014). Resting-state brain activation correlates with short-time antidepressant 
treatment outcome in drug-naive patients with major depressive disorder. J Int Med Res 42: 966-975.

Watters AJ, Williams LM (2011). Negative biases and risk for depression; integrating self-report and emotion task markers. Depress Anxiety 28: 703-718.

Woodward ND, Rogers B, Heckers S (2011). Functional restingstate networks are differentially affected in schizophrenia. Schizophr Res 130: 86-93.

Woolrich MW, Ripley BD, Brady M, Smith SM (2001). Temporal autocorrelation in univariate linear modeling of FMRI data. Neuroimage 14: 1370-1386.

Wu QZ, Li DM, Kuang WH, Zhang TJ, Lui S, Huang XQ et al (2011). Abnormal regional spontaneous neural activity in treatment-refractory depression revealed by resting-state fMRI. Hum Brain Mapp 32: 1290-1299.

Yang R, Zhang H, Wu X, Yang J, Ma M, Gao Y et al (2014). Hypothalamus-anchored resting brain network changes before and after sertraline treatment in major depression. Biomed Res Int 2014: 915026.

Yuen GS, Gunning-Dixon FM, Hoptman MJ, AbdelMalak B, McGovern AR, Seirup JK et al (2014). The salience network in the apathy of late-life depression. Int J Geriatr Psychiatry 29: 1116-1124.

Zakzanis KK, Leach L, Kaplan E (1998). On the nature and pattern of neurocognitive function in major depressive disorder. Neuropsychiatry Neuropsychol Behav Neurol 11: 111-119.

Zhang WN, Chang SH, Guo LY, Zhang KL, Wang J (2013). The neural correlates of reward-related processing in major depressive disorder: a meta-analysis of functional magnetic resonance imaging studies. J Affect Disord 151: 531-539.

Zhu X, Wang X, Xiao J, Liao J, Zhong M, Wang W et al (2012). Evidence of a dissociation pattern in resting-state default mode network connectivity in first-episode, treatment-naive major depression patients. Biol Psychiatry 71: 611-617.

Supplementary Information accompanies the paper on the Neuropsychopharmacology website (http://www.nature.com/npp) 